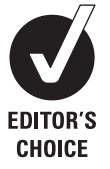

'Department of Medicine 3, Division of Rheumatology, Medical University of Vienna, Vienna, Austria

${ }^{2}$ Second Department of Medicine, Hietzing Hospital, Vienna, Austria

\section{Correspondence to}

Daniel Aletaha, Department of Internal Medicine 3, Division of Rheumatology, Medical University Vienna, Waehringer Guertel 18-20, 1090 Vienna, Austria;

daniel.aletaha@meduniwien. ac.at

Accepted 2 January 2011 Published Online First 14 February 2011

\title{
Physical disability in rheumatoid arthritis is associated with cartilage damage rather than bone destruction
}

\author{
Daniel Aletaha, ${ }^{1}$ Julia Funovits, ${ }^{1}$ Josef S Smolen ${ }^{1,2}$
}

\begin{abstract}
Background Joint destruction in rheumatoid arthritis is comprised of cartilage and bone damage, which can be evaluated radiographically separately by the joint space narrowing (JSN) and erosion (ERO) scores. It is currently unclear to which extent these components affect irreversible functional disability. The aim of the present work was to determine these contributions.

Methods Data, kindly provided by the sponsors, was evaluated from several randomised controlled clinical trials on adalimumab, etanercept, infliximab and leflunomide. Patients who reached stringent remission according to the Simplified Disease Activity Index (SDAI $\leq 3.3)$ were extracted to eliminate the activity related (ie, reversible) component of disability. In these patients, residual Health Assessment Questionnaire Disability Index (HAO-DI) score at the time of remission (to reflect the level of 'irreversible' disability) was determined and related to baseline measures of ERO and JSN scores univariately, by stratification and in adjusted regression models.
\end{abstract}

Results A total of 748 patients who achieved a state of remission were analysed $16.3 \%$ of the total pooled population of 4602 patients). In the univariate analyses, mean residual HAQ-DI values in remission were significantly larger in higher tertiles of JSN and ERO (ERO: 0.21, 0.25, 0.35; JSN: 0.19, 0.24, 0.39; $p<0.001$ for both). In stratified analyses, mean residual HAQ-DI scores were larger in higher tertiles of JSN within the first tertile of ERO $(0.18,0.25,0.29 ; p=0.05)$, as well as the second $(0.21,0.24,0.29 ; p=0.19)$ and the third $(0.12$, $0.23,0.42 ; p<0.001)$. In contrast, there was no such trend across ERO tertiles within the first JSN tertile $(0.18$, $0.21,0.12 ; p=0.99)$ and the second tertile $(0.25,0.24$, $0.23 ; p=0.77)$, and only marginally within the third tertile of JSN (0.29, 0.29, 0.42; $p=0.07)$. Adjusted multivariate regression models supported the significant association of JSN on residual disability.

Conclusions Cartilage damage appears to be the more clearly associated with irreversible physical disability than bony damage. These data suggest that particular attention should be given to therapeutic interference with cartilage destruction.

\section{INTRODUCTION}

Synovitis, joint damage and impairment of physical function are the hallmarks of rheumatoid arthritis (RA). While pathogenetically the influx of immune and inflammatory cells signifies synovitis, its invasion into the adjacent bone and the consumption of cartilage constitute the destructive elements of the disease. Physical disability is then the consequence of the ongoing inflammatory activity (pain, stiffness, swelling, usually assessed by composite disease activity measures) and the destructive events (usually assessed by radiographic scoring). Indeed, deficiency of physical functioning as evaluated by the Health Assessment Questionnaire Disability Index (HAQ-DI) is associated with the extent of disease activity and joint damage. ${ }^{1-3}$ Importantly, though, physical disability is only partly reversible, namely as far as it is mediated by disease activity; its irreversible components are primarily related to joint damage, ${ }^{4}$ although other factors such as comorbidity may also contribute. ${ }^{5}$

Indeed, with increasing joint damage, the extent of physical impairment in the absence of active disease, that is, in remission, also increases. ${ }^{4}$ Likewise, higher short-term progression of joint damage is associated with more disability. ${ }^{6}$ Moreover, the relationship between irreversible disability and joint damage can be estimated and is almost linear over a certain range of the radiographic score. ${ }^{7}$

The two major components of joint damage characteristic of RA, cartilage degradation and bony destruction, are elicited by distinct mechanisms. While the latter is mediated primarily by synovial osteoclasts and direct invasion of the pannus into the adjacent bone, ${ }^{8} 9$ cartilage damage is mostly due to the action of metalloproteinases, secreted into the synovial fluid by synovial fibroblasts and macrophages, or produced locally by activated chondrocytes, events that are governed by proinflammatory cytokines. ${ }^{10} 11$ Cartilage degradation is reflected radiologically by joint space narrowing (JSN) and bony changes by erosions (EROs), ${ }^{12}$ both of which are assessed separately when employing the total Sharp score (TSS) and its modifications. ${ }^{13}$ It has recently been shown that these two events follow different pathomechanisms and can progress independently from each other. ${ }^{14}$ However, in the diagnosis of RA, as well as in the assessment of its radiological progression over time, the focus of attention is often on the erosive process, rather than JSN. ${ }^{15-17}$

Treatment of RA aims at interference with disease activity and joint damage in order to maximise the effects on long-term physical function. However, when addressing the consequences of joint damage on physical disability it is not known at present to which extent the two components, bony and cartilage damage, contribute individually to the irreversible disability shown to be related to joint destruction. The aim of the present study was, therefore, to address the individual contributions of EROs and narrowing to functional loss in patients with RA. 


\section{MATERIALS AND METHODS}

We obtained and pooled individual patient data from several trials: the Antivascular Targeted Therapy: Researching ASA404 in Cancer Treatment (ATTRACT) trial ${ }^{18}$ of infliximab and methotrexate (MTX) versus placebo and MTX in patients with inadequate prior response to MTX; the AutoPulse Assisted Prehospital International Resuscitation (ASPIRE) trial ${ }^{19}$ of infliximab plus MTX versus MTX alone in patients who were MTX naive with early RA of 3 years or less; the DE01920 trial of patients with active disease despite prior MTX therapy receiving additional adalimumab versus placebo; the PREMIER trial of adalimumab plus MTX in comparison with either monotherapy in patients with early arthritis of 3 years or less ${ }^{21}$; the Trial of Etanercept and Methotrexate with Radiographic Patient Outcomes (TEMPO) study of etanercept plus MTX versus either alone in patients with established $\mathrm{RA}^{22}$; and the European leflunomide trials, which compared leflunomide with sulfasalazine and with placebo in patients with early and established RA, ${ }^{23} 24$ or compared leflunomide with MTX in patients with 10 years of RA or less. ${ }^{25}$ We were provided data on an $80 \%$ to $90 \%$ random sample of subjects from these trials. All patients had active RA at enrolment into these studies, with requirements for more than 6-10 swollen joints and more than 6-12 tender joints (using a 68-joint count), or 6 or more swollen and 6 or more tender joints (using a 28 -joint count) in the leflunomide trials. With the exception of the ASPIRE trial, elevations in acute phase reactants were required in all trials (C reactive protein $\geq 1.5$ or $2.0 \mathrm{mg} / \mathrm{dl}$ and/or erythrocyte sedimentation rate $\geq 28 \mathrm{~mm} / \mathrm{h}$ ). Data on scored radiographs using the TSS $^{26}$ (DE019, ERA, PREMIER and Leflunomide trials) or van der Heijde modified TSS ${ }^{27}$ (ASPIRE, ATTRACT and TEMPO trials) were available for all trials and included the details on JSN and ERO scores. The TSS and its modification generally produce very similar results, but can be different in patients with extensive damage of joints in the feet. To diminish these differences, we defined the subgroups for analysis based on the distribution of the respective scores within each trial using tertiles. Only patients in whom all data needed for the present analysis, that is, Simplified Disease Activity Index (SDAI), HAO-DI and TSS with ERO and JSN scores, were available were assessed.

\section{Study variables}

In each trial, the HAQ-DI was used as a measure of limitations of physical function. ${ }^{15}$ It includes 20 questions that are organised in 8 categories (dressing, rising, eating, walking, hygiene, reach, grip and errands or chores). Each question is scored using a fourlevel scale ranging from 0 to 3 , representing no difficulty (' 0 '), some difficulty (' 1 '), much difficulty (' 2 ') and unable to do (' 3 '). The HAQ-DI score is the mean of the highest score in each of the eight categories, with a possible scoring range of $0-3$. To determine the irreversible HAQ-DI, we obtaining the HAQ-DI score at the time of clinical remission (see below).

We assessed the JSN and ERO scores as variables reflecting cartilage and bone damage, respectively. Since our investigation related to the association of joint damage with irreversible physical disability and this did not depend on potentially different efficacy of the types of therapy, we pooled data from all treatment arms to maximise the statistical power.

\section{Definition of irreversible functional disability}

To separate the reversible, RA activity related component of disability from the irreversible component, we identified patients who achieved clinical remission during the trials, that is, a state of absence of significant RA activity and thus absence of a reversible disability component.

We defined remission as a state below a threshold value of a composite measure of RA activity, the $\mathrm{SDAI}^{28}$ : $\mathrm{SDAI}=\mathrm{SJC} 28+\mathrm{TJC} 28+\mathrm{PGA}+\mathrm{EGA}+\mathrm{CRP}$, where SJC28 and TJC28 denote swollen or tender joints using the 28-joint count, EGA is evaluator global assessment of disease activity and PGA is patient global assessment of disease activity (both using a 10 $\mathrm{cm}$ visual analogue scale), and CRP is C reactive protein in $\mathrm{mg} /$ dl. This index has been repeatedly validated ${ }^{29-31}$ and provides a stringent remission cutpoint, $\mathrm{SDAI} \leq 3.3 .{ }^{32-35}$ Indeed, the majority of patients below this cutpoint do not show progression of radiographic damage over time. ${ }^{36}$

We identified patients achieving clinical remission by SDAI at the 3-month, 6-month, 9-month or 12-month follow-up visits in the trials. Each patient's first visit in remission was used for analysis. The HAQ-DI score at this visit was considered to be the best estimate of a patient's irreversible functional disability.

\section{Analysis}

We tested the association of JSN and EROs (using the respective tertiles) with the amount of irreversible functional disability (as continuous variable) using simple univariate analysis, stratified analysis and adjusted multivariate models. In the univariate analysis, we calculated means and $95 \%$ CIs of irreversible HAQ-DI and used one-way analysis of variance testing for a linear trend component, as the statistical test.

In the stratified approach, we performed separate analyses of the effects of JSN (tertiles) within the respective strata of EROs (tertiles), and vice versa.

Finally, we used a multivariate regression model (generalised linear model (GLM)) to investigate the independent statistical association of EROs and JSN (included as continuous variables), adjusted for additional potential confounders, namely SDAI, age and duration of RA with irreversible function. Nevertheless, given the large number of patients involved and the central limit theorem, the use of parametric methods would still be legitimate, especially in a supportive analysis such as this one (with the main analysis being the stratified analysis). We therefore used the GLM to estimated marginal means (EMMs) for independent effects of the two variables, which can be compared in a meaningful way given the absence of transformation of the dependent variables. Disease activity adjustment was done, although the definition of remission employed here (SDAI $\leq 3.3$ ) was very stringent and allows only little variation in disease activity. However, theoretically there could still be differences in residual disease activity (eg, SDAI 3.2 in one individual and SDAI 0.5 in another), and consequently, differences in the amount of disability related to disease activity (which would still be reversible).

From this model, we calculated the independent effects of ERO or JSN and their significances. We then calculated the EMMs (adjusted means with fixed covariate levels) of irreversible HAQ-DI scores for each tertile of JSN setting the continuous variables age, duration of RA and ERO score to their respective (rounded) cohort means, and SDAI to a value of 1.0, corresponding to solid remission (providing best estimation of irreversible damage). We then calculated the EMMs for tertiles of ERO in the same way, setting age, duration and JSN score to the means, and SDAI to 1.

In an additional sensitivity analysis, we calculated the irreversible HAQ-DI scores not as means of their absolute values, but 
rather as the proportion of the respective baseline values (percentage irreversibility of functional disability) in clinical remission, and used this outcome instead of the mean irreversible HAQ-DI. This measure takes the more clinical perspective of estimating the best possible proportional improvement of function in a patient before treatment is initiated. For each patient, we calculated the percentage reversibility of HAQ-DI scores as (change in $\mathrm{HAQ}-\mathrm{DI} /$ baseline HAQ-DI) $\times 100 \%$, and complementary of the irreversibility as $(100 \%$ - percentage reversibility). Since baseline HAQ-DI scores of 0 cannot improve, we excluded such patients from this analysis. We then investigated if percentage irreversibility of HAQ-DI was associated with JSN, ERO, or both, using the Kruskal-Wallis test across the respective tertiles. In another sensitivity analysis we loosened the definition of remission and changed it to low disease activity (LDA) (ie, allowing more reversible disease activity to 'contaminate' the state of irreversible functional disability).

Analyses were performed using SAS software, V.9.2 (SAS Institute, Cary, North Carolina, USA); p values $<0.05$ were regarded as significant.

\section{RESULTS}

\section{Patient demographics}

Data for 4602 patients (pooled total population) were available for the current analysis. Among them, 748 patients attained remission as defined above and their data were used for the current analyses. Baseline demographics of these patients are shown in table 1 ('total' column). At the time of remission, they had a mean $( \pm$ SD) SDAI of $2.1 \pm 0.8$ and a mean HAQ-DI of $0.27 \pm 0.40$.

To address the contribution of cartilage damage and bone destruction to the irreversible impairment of physical function, we formed tertiles of JSN and ERO scores, respectively. The baseline clinical disease characteristics of patients did not differ significantly across the tertiles of JSN or ERO, with the exception of small differences regarding SJC (table 1). However, mean age and mean disease duration were profoundly higher with increasing JSN or ERO score groups, but were not different when the first, second and third tertiles of JSN were compared with the respective tertiles by ERO (table 1). To address the potential influence of disease duration and age on the irreversible HAQ-DI, some of the subsequent analyses were adjusted for these variables (see below).

\section{Univariate analysis of the effects of JSN and ERO on irreversible physical disability}

The residual HAQ-DI in remission increased across tertiles of ERO, amounting to a mean \pm SD of $0.21 \pm 0.34,0.24 \pm 0.35$ and $0.35 \pm 0.47$, respectively, for the first, second and third tertile ( $<<0.0001$; figure 1A: crude analysis). Likewise and even to a numerically greater extent, with increasing tertiles by JSN, residual (irreversible) HAQ-DI scores increased, amounting to $0.18 \pm 0.33,0.24 \pm 0.35$ and $0.38 \pm 0.47$ ( $p<0.0001$; figure $1 \mathrm{~B}$ : crude analysis). Since JSN and ERO frequently occur in combination, the independent effects of either one are difficult to assess by univariate analysis.

\section{Main analysis: stratified analysis of the independent effects of JSN and ERO on irreversible physical disability}

To address the independent contribution of ERO, we looked at the effects of higher ERO tertile within each of the tertile strata by JSN scores. As can be seen in figure $2 \mathrm{~A}$, in the first and second tertiles formed according to JSN, higher degrees of erosive changes did not contribute at all to irreversible disability $(p=0.99$ and 0.77 , respectively). Not unexpectedly, the group of patients in the third tertile of JSN and ERO had the worst functional disability (figure $2 \mathrm{~A}$; $\mathrm{p}$ value across the ERO tertiles $=0.07$ ). This group also includes the patients with truly severe joint damage, its contribution to the trends should therefore be interpreted with caution.

By contrast, JSN did appear to convey irreversible impairment of physical function, when analysed within each tertile by ERO (figure $2 \mathrm{~B}$ ). These trends manifested in all three strata by ERO $(\mathrm{p}<0.05 ; \mathrm{p}=0.19$ and $\mathrm{p}<0.001$, respectively).

\section{Supportive analysis: adjusted analysis of the independent effects of JSN and ERO on irreversible physical disability}

As indicated above, there were significant differences regarding baseline patient age and disease duration between the JSN, or ERO, tertiles. Also, patients in SDAI remission might still have varying underlying SDAI scores (0-3.3), and even in the tertile analysis above patients might still have differences in ERO scores within a particular ERO tertile (eg, ERO scores different in first ERO tertile of JSN-T1, JSN-T2 and JSN-T3).

Therefore, based on GLMs, we used EMMs adjusting for these variables (see Materials and methods section). Figure $1 \mathrm{~A}, \mathrm{~B}$ (adjusted analysis) depict the EMMs of HAQ-DI scores for the

Table 1 Baseline characteristics for the total patient population and for subgroups formed according to tertiles by ERO scores and by JSN scores

\begin{tabular}{|c|c|c|c|c|c|c|c|}
\hline \multirow[b]{2}{*}{ Variable } & \multirow[b]{2}{*}{ Total $(n=748)$} & \multicolumn{3}{|c|}{ Tertile of ERO score } & \multicolumn{3}{|c|}{ Tertile of JSN score } \\
\hline & & $T_{1}(n=247)$ & $T_{2}(n=251)$ & $T_{3}(n=250)$ & $T_{1}(n=258)$ & $T_{2}(n=234)$ & $T_{3}(n=256)$ \\
\hline Age, years & $49.9 \pm 13.3$ & $46.7 \pm 13.4$ & $50.4 \pm 12.3$ & $52.7 \pm 13.4$ & $45.8 \pm 12.6$ & $50.2 \pm 12.3$ & $53.9 \pm 13.6$ \\
\hline Duration, years & $3.1 \pm 5.4$ & $1.7 \pm 2.9$ & $2.2 \pm 3.6$ & $5.4 \pm 7.6$ & $1.4 \pm 2.0$ & $2.1 \pm 3.6$ & $5.7 \pm 7.7$ \\
\hline ERO score, mean $\pm S D$ & $11.9 \pm 18.7$ & $0.6 \pm 0.7$ & $5.6 \pm 2.5$ & $29.4 \pm 23.9$ & $3.1 \pm 4.8$ & $7.6 \pm 8.4$ & $24.7 \pm 26.1$ \\
\hline ERO score, range & $0-169.5$ & $0-2.3$ & $2.5-11.0$ & $11.3-169.5$ & $0-30.8$ & $0-58.8$ & $0-169.5$ \\
\hline Narrowing score, mean $\pm S D$ & $8.0 \pm 15.0$ & $1.8 \pm 4.3$ & $3.9 \pm 6.4$ & $18.3 \pm 21.4$ & $0.1 \pm 0.2$ & $2.6 \pm 1.3$ & $20.9 \pm 20.1$ \\
\hline Narrowing score, range & $0-120.5$ & $0-36.5$ & $0-61.5$ & $0-120.5$ & $0-0.5$ & $0.6-5.4$ & $5.5-120.5$ \\
\hline HAQ-DI & $1.3 \pm 0.6$ & $1.2 \pm 0.6$ & $1.4 \pm 0.6$ & $1.3 \pm 0.6$ & $1.3 \pm 0.6$ & $1.3 \pm 0.6$ & $1.3 \pm 0.6$ \\
\hline SDAI & $37.2 \pm 13.7$ & $35.5 \pm 14.2$ & $39.4 \pm 13.8$ & $36.8 \pm 13.0$ & $36.3 \pm 13.8$ & $37.5 \pm 13.6$ & $38.0 \pm 13.8$ \\
\hline SJC28 & $10.7 \pm 5.5$ & $9.9 \pm 5.5$ & $11.4 \pm 5.6$ & $11.0 \pm 5.2$ & $10.0 \pm 5.2$ & $10.5 \pm 5.4$ & $11.7 \pm 5.7$ \\
\hline TJC28 & $12.8 \pm 6.3$ & $12.5 \pm 6.3$ & $13.5 \pm 6.2$ & $12.4 \pm 6.4$ & $12.8 \pm 6.2$ & $12.6 \pm 6.1$ & $13.0 \pm 6.7$ \\
\hline $\mathrm{PGA} / \mathrm{cm}$ & $5.4 \pm 2.4$ & $5.4 \pm 2.4$ & $5.6 \pm 2.5$ & $5.2 \pm 2.4$ & $5.4 \pm 2.4$ & $5.6 \pm 2.5$ & $5.2 \pm 2.4$ \\
\hline $\mathrm{EGA}, \mathrm{cm}$ & $5.7 \pm 1.9$ & $5.4 \pm 2.2$ & $6.1 \pm 1.8$ & $5.7 \pm 1.7$ & $5.6 \pm 2.1$ & $5.8 \pm 1.9$ & $5.8 \pm 1.7$ \\
\hline CRP, mg/dl & $2.6 \pm 3.1$ & $2.3 \pm 3.2$ & $2.8 \pm 3.5$ & $2.5 \pm 2.5$ & $2.5 \pm 3.2$ & $2.9 \pm 3.4$ & $2.3 \pm 2.5$ \\
\hline
\end{tabular}

Bold font denotes significant differences for the indicated variables among tertiles formed by JSN or EROs; these differences were all $p<0.0001$ except for SJC among ER0 tertiles (for which it was 0.025)

CRP, C reactive protein; EGA, evaluator global assessment of disease activity; ERO, erosion; HAQ-DI, Health Assessment Questionnaire Disability Index score; JSN, joint space narrowing; PGA, patient global assessment of disease activity; SDAI, Simplified Disease Activity Index; SJC, swollen joint count; TJC, tender joint count. 

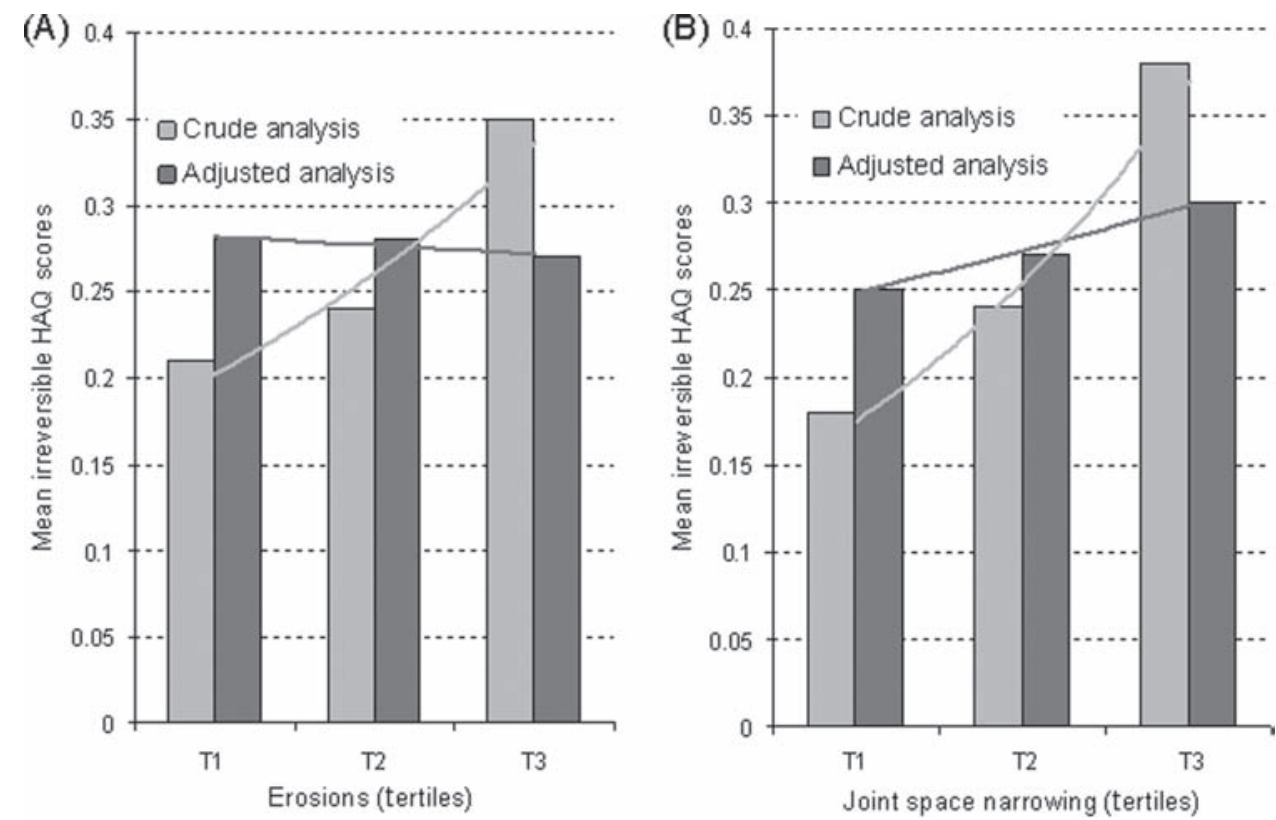

Figure 1 Crude and adjusted analysis of the effects of erosions (ERO) and joint space narrowing (JSN) on irreversible functional disability. (A) Effects of ERO: light grey bars show the crude analysis of ERO depicting the mean irreversible Health Assessment Questionnaire Disability Index (HAO-DI) score in each tertile of ERO ( $p<0.001$ by analysis of variance); dark grey bars show the adjusted analysis depicting estimated marginal means of irreversible HAQ-DI scores for an average patient (with 49.9 years of age, 3.1 years of rheumatoid arthritis duration and a JSN score of 8.0) in strict remission (Simplified Disease Activity Index (SDAI) of 1.0); adjusted $p=0.63$ for ERO. (B) Effects of JSN: Crude analysis (light grey bars; $\mathrm{p}<0.001$ ) and adjusted (dark grey bars) analysis of JSN effects on irreversible HAQ-DI. For the adjusted analysis, the same assumptions were made as in the ERO analysis above, except a cohort mean of ERO of 11.9 was used for adjustment; the adjusted $p<0.001$ for JSN.

three tertiles of ERO and JSN, respectively, at fixed levels of the covariates. It can be seen that the clear associations between level of erosiveness and poorer physical function disappeared after adjustment for the covariates in the model $(p=0.63)$, while the associations for JSN were toned down, but remained highly significant $(\mathrm{p}<0.001)$. Age and duration both contributed independently and significantly in this model $(p<0.001$ and $p=0.002$, respectively), while, not unexpectedly, SDAI values in these patients in remission (SDAI $<3.3$ ) were not significant $(\mathrm{p}=0.87)$. Finally, we performed an analysis using tertiles of ERO and JSN, as well as their interaction term in the same model. We found no significant interaction $(\mathrm{p}=0.26)$. Analysing ERO score and JSN separately in a multivariate analysis with adjustment for age, disease duration and gender, revealed a significant independent contribution of JSN $(\mathrm{p}=0.003)$ but not of ERO score $(\mathrm{p}=0.95)$ in explaining variation in irreversible physical disability.

\section{Sensitivity analysis}

In addition to the above analysis of absolute (mean) irreversible functional disability, we also analysed irreversible disability expressed as proportion of baseline HAO-DI. The effect of JSN was again apparent in this analysis (figure 3B). In contrast, higher ERO scores again clearly did not increase this proportion independently of JSN scores (figure $3 \mathrm{~A}$ ).

We finally evaluated the contributions of ERO and JSN among patients in $\mathrm{LDA}$ (SDAI $>3.3$ to 11 ) rather than remission. Although in these patients a considerably higher amount of reversible disability (mediated through disease activity) is present, the results of this analysis fully confirmed the above findings. For example, within the first JSN tertile, residual HAQ-DI levels were $0.47 \pm 0.49,0.51 \pm 0.47$ and $0.45 \pm 0.53$ for the first, second and third ERO tertile, respectively ( $\mathrm{p}=$ not significant). In contrast, within the first ERO tertile residual HAO-DI values amounted to $0.47 \pm 0.49,0.55 \pm 0.55$ and $0.73 \pm 0.60$ for the three JSN tertiles, respectively $(\mathrm{p}<0.0001)$. Similar observations were made for the respective second and third tertiles (data not shown). These data confirmed the major contribution of JSN rather than ERO to disability.

\section{DISCUSSION}

Joint damage is a pivotal characteristic of RA and consists of cartilage degradation and bone destruction, which can be visualised radiologically as JSN and EROs. However, EROs are regarded prototypic for RA, as also indicated by the radiographic criteria of the 1987 RA classification criteria, while JSN is seen as less characteristic, since it can occur in a variety of other disorders, including reactive arthritis or degenerative joint disease. ${ }^{15-17} 37$ Nevertheless, despite the specificity of erosive bone damage for the entity RA, in the present study we have been able to show that for the clinical impact of joint destruction cartilage loss seems to be of much higher relevance than damage to the bone.

We used physical disability as the measure of 'clinical impact', and HAQ-DI scores as the instrument to quantify it. However, limitations of physical functioning are complex by their nature, and are comprised of reversible as well as irreversible components. Indeed, pain and stiffness as reflection of disease activity can impair functional capacity, but this impairment is reversible with remission of disease activity. ${ }^{4}$ In contrast, increasing joint damage leads to increasing irreversible disability. ${ }^{4}$ This irreversible disability is highly relevant, as it is no longer amenable to treatment, even with the most intensive therapies available. ${ }^{38}$

It has been shown in the past that joint damage (as assessed by radiographic evaluation) increases functional disability, ${ }^{1} 4$ and this was also confirmed in the present study. We have recently devised a method to roughly estimate the functional impact of increases in radiographic damage, ${ }^{7}$ but until now the 

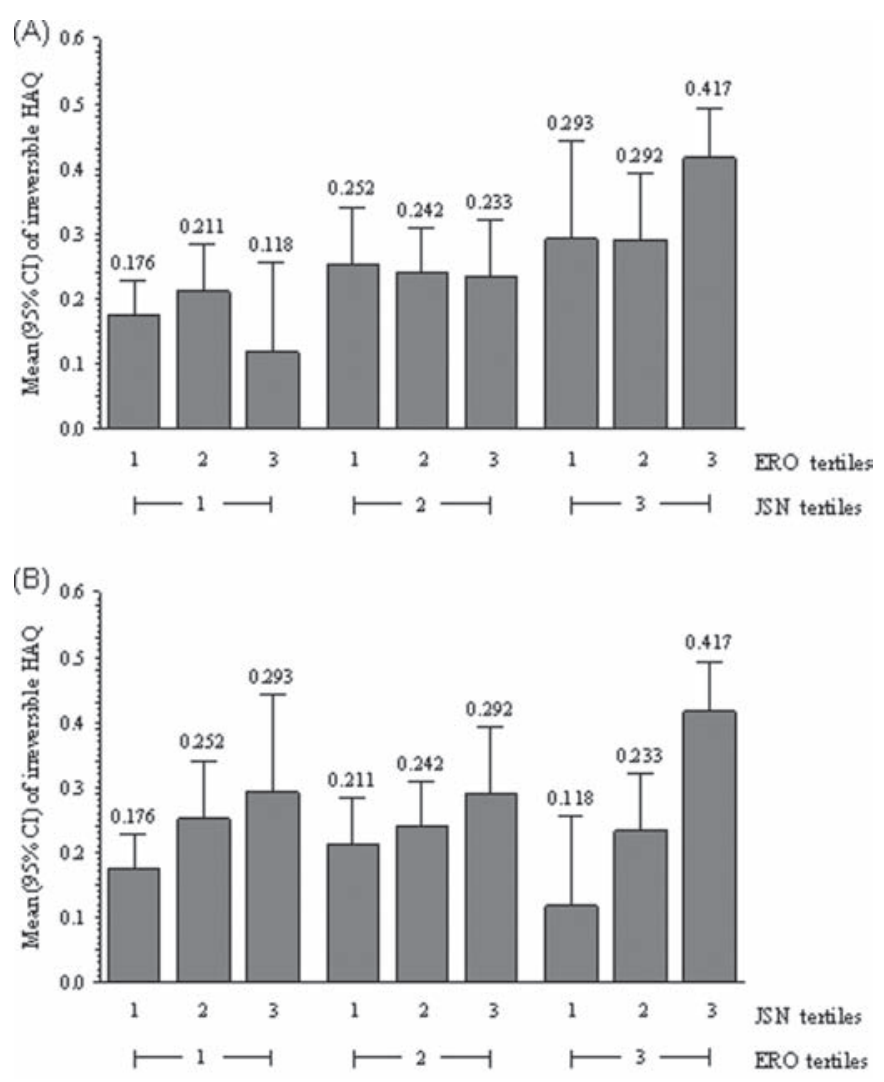

Figure 2 Stratified analysis on the effects of erosions (ERO) and joint space narrowing (JSN) on irreversible Health Assessment Questionnaire Disability Index (HAQ-DI) scores (mean + upper limit of 95\% CI for HAQ-DI score in Simplified Disease Activity Index (SDAl) remission). (A) Effects of ERO: bars arranged according to clusters by tertiles of JSN $\left(T_{1}, T_{2}, T_{3}\right)$ to assess the effects of ERO $(p=0.99,0.77$ and 0.07 , respectively, for $T_{1}, T_{2}, T_{3}$, using analysis of variance testing for linear trend component). (B) Effects of JSN: bars arranged according to clusters by tertiles of ERO to assess the effects of JSN (the p values for the effects of JSN were $0.05,0.19,<0.001$, respectively, within each of the ERO tertiles).

distinction and relevance of cartilage and bony damage has not been considered in measurement aspects and therapy. A look at the impact of these radiological features separately revealed that cartilage damage is clearly more relevant than bony damage when function is considered.

Undoubtedly, joint damage is just one aspect among many determining functional loss, even in clinical remission, and much of the variability seen in disability is explained by other causes, or simply remains unexplained. We therefore used all measured potential confounders in an adjusted multivariate model considering age, disease duration and disease activity, in addition to the two features of joint destruction. Also, in these adjusted models JSN showed a highly significant independent effect, which was in contrast to the lacking effect observed for EROs. Additional sensitivity analysis further strengthened the results of our study.

One limitation of our study is the insufficient ability to visualise the inside of the joint, since radiographic changes are only a surrogate of the joint pathology. Moreover, the scoring system may be too crude and differs inherently for JSN versus EROs. ${ }^{26} 39$ Nevertheless, radiographic joint damage in general and JSN and ERO scores in particular have been shown repeatedly to be valid measures that are related to disease activity and physical
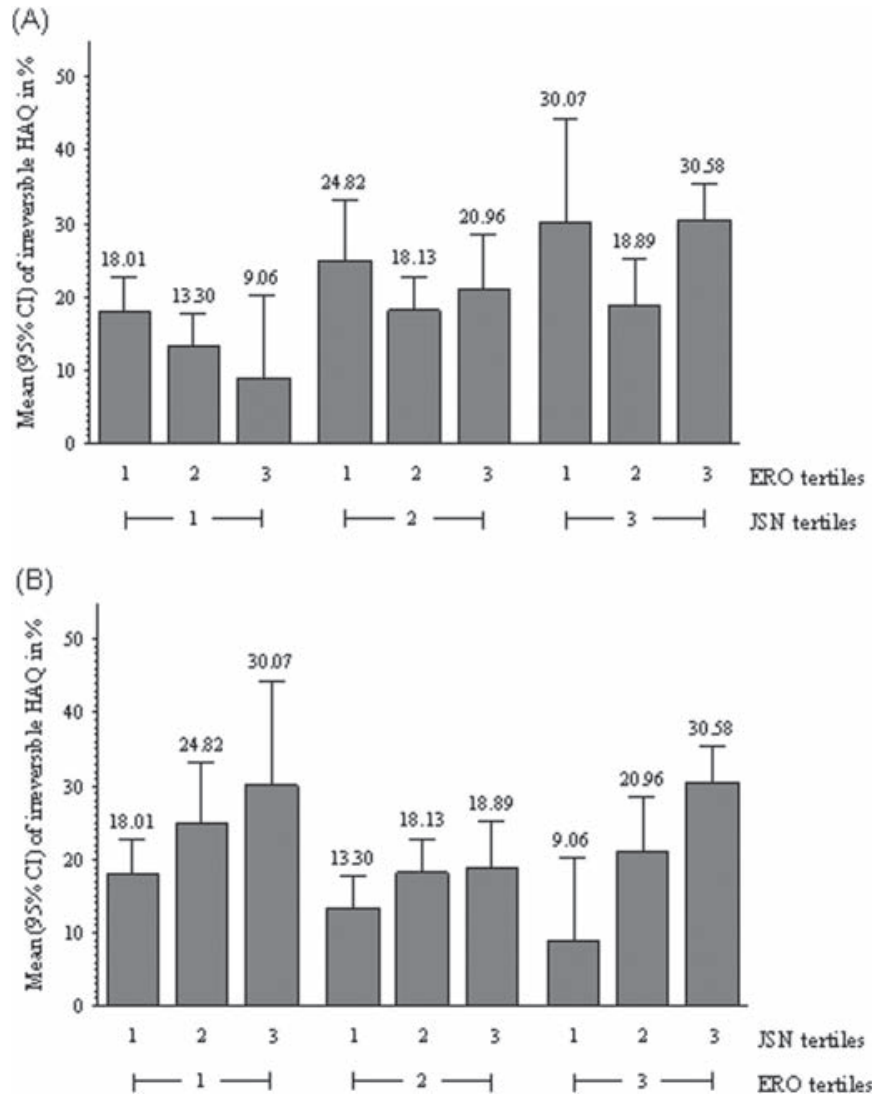

Figure 3 Stratified analysis using percentage irreversible functional disability as the outcome (expressed as a fraction: disability observed in remission/disability at baseline $\times 100$ ). (A) Effects of erosions (ERO): bars arranged according to clusters by tertiles of joint space narrowing (JSN; $\left.T_{1}, T_{2}, T_{3}\right)$ to assess the effects of ERO $\left(T_{1}, T_{2}, T_{3}\right)$; the $p$ values for the effects of ERO were $0.09,0.43$ and 0.17 , respectively within the strata of JSN tertiles (analysis of variance testing for linear trend component across ERO tertiles). (B) Effects of JSN: bars arranged according to clusters by tertiles of ERO to assess the effects of JSN ( $p=0.04,0.13,<0.001$, respectively).

function. Moreover, the fact that a compound targeting osteoclast activation, denosumab, was effective in reducing progression of erosive joint damage but not $\mathrm{JSN}^{40}$ confirms the validity of the assumption that JSN reflects cartilage damage and that the mechanisms leading to this type of damage differ from those operative in the process of EROs.

Another limitation of our study may be that we possibly did not assess patients with maximal reduction of disease activity related impairment of physical function, since we identified remission at its first occurrence and did not assess patients with persistent remission nor used additional imaging techniques to identify subclinical synovitis. However, given that the sensitivity analysis in patients with LDA resulted in similar observations, a potential small residual disease activity in clinical remission is unlikely to have played a major role. Also, by using this approach we were able to increase the sample size. A strength of the study is the use of a large sample size from several randomised controlled clinical trials with complete data, which was key to the performance of this study in a very stringently defined subgroup of patients in SDAI remission. However, patient populations in trials are selected for various characteristics. Therefore, it would be good to have a validation of our findings also in observational cohorts, where for example patients with relevant comorbidities are also represented. 
It would not have been possible to obtain the results presented if a global joint damage score, such as the Larsen score, ${ }^{41}$ had been employed. Thus, our findings also imply that it may be necessary to evaluate these two components separately in routine clinical follow-up of patients with RA in order to fully appreciate the consequences of their joint damage.

It is important to emphasise that the present study is a crosssectional association study on the effects of EROs and JSN on physical function in RA. Although this is the first study to test this association, we cannot conclude that an increase over time in EROs or JSN in fact leads to impairment of function. For this purpose study designs different from the present one with longitudinal data analysis need to be performed. Nevertheless, the data presented here provide sufficient evidence for developing, and further testing of, the hypothesis that cartilage damage rather than bone destruction may constitute the pivotal cause of physical functional impairment in RA. Further proof of this hypothesis may suggest that therapeutic interventions in RA ought to interfere with the totality of joint damage and particularly with cartilage degradation as reflected by JSN. At the same time, JSN might be considered the better surrogate of overall joint damage than erosiveness. Fortunately, synthetic and biological disease-modifying antirheumatic drugs licensed today for the treatment of RA affect joint damage in its totality and do not spare one of the two components. ${ }^{19} 2122$ 42-45 Importantly, however, since even relatively little cartilage degradation can lead to significant impairment of physical functioning, early therapeutic intervention to prevent occurrence of such damage is of particular importance.

Acknowledgements We thank Abbott, Amgen, Centocor, Sanofi-Aventis and Wyeth, which was acquired by Pfizer in October 2009, for kindly providing the data from their clinical trials. This study was supported through Coordination Theme 1 (Health) of the European Community's FP7; Grant Agreement number HEALTH-F2-2008-223404 (Masterswitch). This is a publication of the Joint and Bone Center for Diagnosis, Research and Therapy of Musculoskeletal Disorders of the Medical University of Vienna.

Competing interests None.

Provenance and peer review Not commissioned; externally peer reviewed.

\section{REFERENCES}

1. Drossaers-Bakker KW, de Buck M, van Zeben D, et al. Long-term course and outcome of functional capacity in rheumatoid arthritis: the effect of disease activity and radiologic damage over time. Arthritis Rheum 1999;42:1854-60.

2. Welsing PM, van Gestel AM, Swinkels HL, et al. The relationship between disease activity, joint destruction, and functional capacity over the course of rheumatoid arthritis. Arthritis Rheum 2001:44:2009-17.

3. Smolen JS, Aletaha D. Patients with rheumatoid arthritis in clinical care. Ann Rheum Dis 2004;63:221-5.

4. Aletaha D, Smolen J, Ward MM. Measuring function in rheumatoid arthritis: identifying reversible and irreversible components. Arthritis Rheum 2006;54:2784-92.

5. Radner H, Smolen JS, Aletaha D. Impact of comorbidity on physical function in patients with rheumatoid arthritis. Ann Rheum Dis 2010;69:536-41.

6. van der Heijde D, Landewé $R$, van Vollenhoven $R$, et al. Level of radiographic damage and radiographic progression are determinants of physical function: a longitudinal analysis of the TEMPO trial. Ann Rheum Dis 2008;67:1267-70.

7. Smolen JS, Aletaha D, Grisar JC, et al. Estimation of a numerical value for joint damage-related physical disability in rheumatoid arthritis clinical trials. Ann Rheum Dis 2010;69:1058-64.

8. Gravallese EM, Harada Y, Wang JT, et al. Identification of cell types responsible for bone resorption in rheumatoid arthritis and juvenile rheumatoid arthritis. Am J Pathol 1998;152:943-51.

9. Redlich K, Hayer S, Ricci R, et al. Osteoclasts are essential for TNF-alpha-mediated joint destruction. J Clin Invest 2002;110:1419-27.

10. Goldring SR. Pathogenesis of bone and cartilage destruction in rheumatoid arthritis. Rheumatology (Oxford) 2003;42(Suppl 2):ii11-16.

11. Milner JM, Cawston TE. Matrix metalloproteinase knockout studies and the potential use of matrix metalloproteinase inhibitors in the rheumatic diseases. Curr Drug Targets Inflamm Allergy 2005;4:363-75.

12. Verstappen SM, Poole AR, lonescu M, et al. Radiographic joint damage in rheumatoid arthritis is associated with differences in cartilage turnover and can be predicted by serum biomarkers: an evaluation from 1 to 4 years after diagnosis. Arthritis Res Ther 2006;8:R31.

13. Sharp JT, Lidsky MD, Collins LC, et al. Methods of scoring the progression of radiologic changes in rheumatoid arthritis. Correlation of radiologic, clinical and laboratory abnormalities. Arthritis Rheum 1971;14:706-20.

14. Smolen JS, van der Heijde DM, Aletaha D, et al. Progression of radiographic joint damage in rheumatoid arthritis: independence of erosions and joint space narrowing. Ann Rheum Dis 2009;68:1535-40.

15. Arnett FC, Edworthy SM, Bloch DA, et al. The American Rheumatism Association 1987 revised criteria for the classification of rheumatoid arthritis. Arthritis Rheum 1988;31:315-24.

16. Aletaha D, Neogi T, Silman AJ, et al. 2010 rheumatoid arthritis classification criteria: an American College of Rheumatology/European League Against Rheumatism collaborative initiative. Ann Rheum Dis 2010;69:1580-8.

17. Aletaha D, Neogi T, Silman AJ, et al. 2010 Rheumatoid arthritis classification criteria: an American College of Rheumatology/European League Against Rheumatism collaborative initiative. Arthritis Rheum 2010;62:2569-81.

18. Lipsky PE, van der Heijde DM, St Clair EW, et al. Infliximab and methotrexate in the treatment of rheumatoid arthritis. Anti-Tumor Necrosis Factor Trial in Rheumatoid Arthritis with Concomitant Therapy Study Group. N Engl J Med 2000;343:1594-602.

19. St Clair EW, van der Heijde DM, Smolen JS, et al. Combination of infliximab and methotrexate therapy for early rheumatoid arthritis: a randomized, controlled trial. Arthritis Rheum 2004;50:3432-43.

20. Keystone EC, Kavanaugh AF, Sharp JT, et al. Radiographic, clinical, and functional outcomes of treatment with adalimumab (a human anti-tumor necrosis factor monoclonal antibody) in patients with active rheumatoid arthritis receiving concomitant methotrexate therapy: a randomized, placebo-controlled, 52-week trial. Arthritis Rheum 2004;50:1400-11.

21. Breedveld FC, Weisman MH, Kavanaugh AF, et al. The PREMIER study: a multicenter randomized, double-blind clinical trial of combination therapy with adalimumab plus methotrexate versus methotrexate alone or adalimumab alone in patients with early, aggressive rheumatoid arthritis who had not had previous methotrexate treatment. Arthritis Rheum 2006;54:26-37.

22. Klareskog L, van der Heijde D, de Jager JP, et al. Therapeutic effect of the combination of etanercept and methotrexate compared with each treatment alone in patients with rheumatoid arthritis: double-blind randomised controlled trial. Lancet 2004;363:675-81.

23. Smolen JS, Kalden JR, Scott DL, et al. Efficacy and safety of leflunomide compared with placebo and sulphasalazine in active rheumatoid arthritis: a doubleblind, randomised, multicentre trial. European Leflunomide Study Group. Lancet 1999:353:259-66.

24. Scott DL, Smolen JS, Kalden JR, et al. Treatment of active rheumatoid arthritis with leflunomide: two year follow up of a double blind, placebo controlled trial versus sulfasalazine. Ann Rheum Dis 2001;60:913-23.

25. Emery $\mathbf{P}$, Breedveld FC, Lemmel EM, et al. A comparison of the efficacy and safety of leflunomide and methotrexate for the treatment of rheumatoid arthritis. Rheumatology (Oxford) 2000;39:655-65

26. Sharp JT, Young DY, Bluhm GB, et al. How many joints in the hands and wrists should be included in a score of radiologic abnormalities used to assess rheumatoid arthritis? Arthritis Rheum 1985;28:1326-35.

27. van der Heijde D. How to read radiographs according to the Sharp/van der Heijde method. J Rheumatol 1999;26:743-5.

28. Smolen JS, Breedveld FC, Schiff MH, et al. A simplified disease activity index for rheumatoid arthritis for use in clinical practice. Rheumatology (Oxford) 2003;42:244-57.

29. American College of Rheumatology Committee to Reevaluate Improvement Criteria. A proposed revision to the ACR20: the hybrid measure of American College of Rheumatology response. Arthritis Rheum 2007;57:193-202.

30. Soubrier M, Zerkak D, Gossec L, et al. Which variables best predict change in rheumatoid arthritis therapy in daily clinical practice? J Rheumato/ 2006;33:1243-6.

31. Gülfe A, Geborek P, Saxne T. Response criteria for rheumatoid arthritis in clinical practice: how useful are they? Ann Rheum Dis 2005;64:1186-9.

32. Aletaha D, Ward MM, Machold KP, et al. Remission and active disease in rheumatoid arthritis: defining criteria for disease activity states. Arthritis Rheum 2005:52:2625-36.

33. Mierau M, Schoels M, Gonda G, et al. Assessing remission in clinical practice. Rheumatology (Oxford) 2007;46:975-9.

34. Balsa A, de Miguel E, Castillo C, et al. Superiority of SDAl over DAS-28 in assessment of remission in rheumatoid arthritis patients using power Doppler ultrasonography as a gold standard. Rheumatology (Oxford) 2010;49:683-90.

35. Linde L, Sørensen J, Østergaard M, et al. Does clinical remission lead to normalization of EQ-5D in patients with rheumatoid arthritis and is selection of remission criteria important? J Rheumatol 2010;37:285-90.

36. Aletaha D, Funovits J, Breedveld FC, et al. Rheumatoid arthritis joint progression in sustained remission is determined by disease activity levels preceding the period of radiographic assessment. Arthritis Rheum 2009;60:1242-9.

37. Jacobson JA, Girish G, Jiang $Y$, et al. Radiographic evaluation of arthritis: inflammatory conditions. Radiology 2008;248:378-89. 
38. Aletaha D, Strand V, Smolen JS, et al. Treatment-related improvement in physical function varies with duration of rheumatoid arthritis: a pooled analysis of clinical trial results. Ann Rheum Dis 2008:67:238-43.

39. van der Heijde DM, van't Hof M, van Riel PL, et al. Development of a disease activity score based on judgment in clinical practice by rheumatologists. J Rheumatol 1993;20:579-81.

40. Cohen SB, Dore RK, Lane NE, et al. Denosumab treatment effects on structural damage, bone mineral density, and bone turnover in rheumatoid arthritis: a twelve-month, multicenter, randomized, double-blind, placebo-controlled, phase II clinical trial. Arthritis Rheum 2008;58:1299-309.

41. Larsen A. How to apply Larsen score in evaluating radiographs of rheumatoid arthritis in long-term studies. J Rheumatol 1995;22:1974-5.

42. Sharp JT, Strand V, Leung H, et al. Treatment with leflunomide slows radiographic progression of rheumatoid arthritis: results from three randomized controlled trials of leflunomide in patients with active rheumatoid arthritis. Leflunomide Rheumatoid Arthritis Investigators Group. Arthritis Rheum 2000;43:495-505

43. Cohen SB, Emery P, Greenwald MW, et al. Rituximab for rheumatoid arthritis refractory to anti-tumor necrosis factor therapy: results of a multicenter, randomized, double-blind, placebo-controlled, phase III trial evaluating primary efficacy and safety at twenty-four weeks. Arthritis Rheum 2006;54:2793-806.

44. Kremer JM, Genant HK, Moreland LW, et al. Effects of abatacept in patients with methotrexate-resistant active rheumatoid arthritis: a randomized trial. Ann Intern Med 2006:144:865-76.

45. Nishimoto N, Hashimoto J, Miyasaka N, et al. Study of active controlled monotherapy used for rheumatoid arthritis, an IL-6 inhibitor (SAMURAI): evidence of clinical and radiographic benefit from an $\mathrm{x}$ ray reader-blinded randomised controlled trial of tocilizumab. Ann Rheum Dis 2007;66:1162-7. 


\section{Correction}

Aletaha D, Funovits J, Smolen JS. Physical disability in rheumatoid arthritis is associated with cartilage damage rather than bone destruction. Ann Rheum Dis 2011;70:733-39. doi:10.1136/ ard.2010.138693. The name of the trials, ATTRACT and ASPIRE were published incorrectly. The correct names should be: ATTRACT: Anti-TNF Trial in Rheumatoid Arthritis with Concomitant Therapy. ASPIRE: Active-controlled Study of Patients receiving Infliximab for the treatment of Rheumatoid arthritis of Early onset. We apologise for this error and for any inconvenience this may have caused. 